\title{
An Investigation of Dipeptides Containing Polar and Nonpolar Side Groups by Curie-Point Pyrolysis Tandem Mass Spectrometry
}

\author{
Alexander S. Noguerola, B. Murugaverl, and Kent J. Voorhees \\ Department of Chemistry and Geochemistry, Colorado School of Mines, Golden, Colorado, USA
}

Methionyl-leucine, leucyl-methionine, phenylalanyl-leucine, and leucyl-phenylalanine have been analyzed to determine dipeptide fragmentation mechanisms in Curie-point pyrolysis tandem mass spectrometry. Results show that fragmentations of dipeptides follow two general pathways, one involving direct cleavage of the dipeptide and the other involving cyclization of the dipeptide. Unique products and strong changes in relative mass spectral peak intensities arise, depending on constituent amino acid residues and their sequence. Also, the length and nature of the side groups strongly direct fragmentation. From these results, the major peaks in the spectra of eight other dipeptides could be readily explained; this suggests that a significant number of dipeptides follow the same general fragmentation mechanisms. (J Am Soc Mass Spectrom 1992, 3, 750-756)

L imited studies have been done on pyrolysis electron ionization mass spectrometry (Py-MS) of dipeptides [1,2], although there is considerable literature on their electron ionization (EI) mass spectra [3-6]. Most of the EI studies, however, are on derivatized compounds, which can have fragmentation pathways significantly altered from the underivatized dipeptides. Two general pathways for dipeptide fragmentation have been commonly proposed or stated: either dipeptide cleavage or dipeptide cyclization and then cleavage. Isotopic labeling has been often used as evidence to support these postulated fragmentation pathways.

Thermally volatilized dipeptides have commonly been found to expel water as the dipeptide cyclizes to form a substituted diketopiperazine $[3,6]$ (henceforth, cyclo-dipeptide [3]). It has also been suggested that dehydration may occur such that an allene results [7], but this is probably unlikely from energetic and steric considerations. Svec and Junk [3], who have published the most comprehensive investigation on EI of heated dipeptides, found that spectra of dipeptides were additive spectra of the dipeptide and the cyclo-dipeptide. They believed that breakup of cyclo-dipeptides occurred in a manner that required retention of the amino acid residues' side groups, 1 , on the cyclic fragments, rather than possible loss of a residue side

Address reprint requests to Kent J. Voorhees, Department of Chemistry and Geochemistry, Colorado School of Mines, Golden, $\mathrm{CO} 80401$. group before ring cleavage as shown in reaction 1:<smiles>[R]C(NC)C(C)=O</smiles>

Structure 1

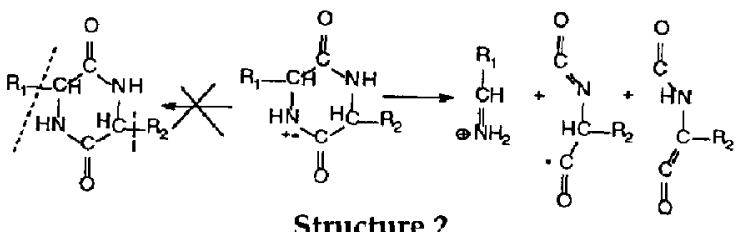

Unfortunately, Svec and Junk [3] thoroughly investigated only cyclo-glycyl-glycine (cyclo(GLY-GLY)) and cyclu-alanyl-alanine (cyclo(ALA-ALA)) fragmentations. Gross and Grodsky [8] and Hurd [9] also noted cyclization of dipeptides upon heating, although their studies did not involve mass spectrometry.

A number of researchers $[1,2,5,10]$ have applied Py-GC/MS to dipeptide analysis and found the same cyclization to occur. Smith et al. [2] stated that dipeptides containing proline yielded exclusively cyclo-dipeptides with some thermal rearrangement of residue side groups. Ratcliff et al. [10] speculated from Py- 
GC/MS results on alanyl-alanine and alanine oligomers that dipeptides formed cyclo-dipeptides and then underwent EI of the cyclo-dipeptide with retention of the side groups on the fragments.

Not everyone who has studied dipeptides has observed formation of the cyclo-dipeptide. Lubman and Li Liang [11] did not observe cyclization upon laser desorption and photon ionization of tyrosine-containing dipeptides. Merritt and Robertson [1] did not observe cyclo-dipeptides as pyrolysis products for $\mathrm{Py}$ GC/MS of glycine dipeptides, but mostly acetone.

Electron ionization cleavage of the dipeptide without formation of a cyclo-dipeptide can also occur. Junk and Svec [4] state that the amino group of the N-terminal residue is ionized and then the $\mathrm{N}$-terminal is cleaved from the dipeptide backbone at the bond $\beta$ to the amino group. They also detected a dipeptide molecular ion peak. Biemann [5] noted additional rearrangements, such as $\gamma$-hydrogen migration to a carbonyl oxygen, 3 .

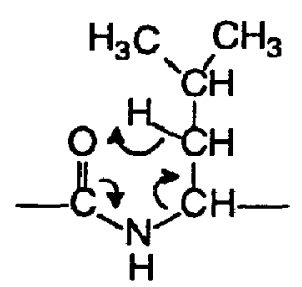

Structure 3

Others [5, 12] have noted that EI of peptides often results in cleavage of the peptide linkage on either side of the carbonyl group, 4.

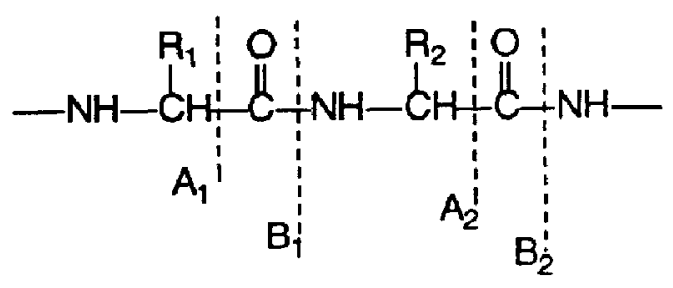

Structure 4

The present paper presents a comprehensive investigation by Curie-point tandem mass spectrometry of 12 dipeptides, which provides insight into the influence of the amino acid residue and its location on dipeptide fragmentation. Although pyrolysis of several different dipeptides have previously been reported, pyrolysis tandem mass spectrometry (Py-MS/MS) provides unique capabilities in determining the sequence of reactions in the general fragmentation pathways.

\section{Experimental}

All spectra were collected on an Extrel model EL-400 (Pittsburgh, PA) triple quadrupole mass spectrometer modified for Curie-point pyrolysis [13]. Triplicate Py-
MS spectra for each dipeptide over the mass range $m / z$ 35 to $\mathbf{3 5 0}$ were collected in random order. Product- and precursor-ion scans were collected in duplicate. All spectra were averaged from at least 20 scans. All spectra were obtained using EI at $70 \mathrm{eV}$. The ion source temperature was $250^{\circ} \mathrm{C}$. Argon was used as the collision gas at a pressure of about 1 torr in the collision cell.

Dipeptide samples were prepared as methanol solutions or suspensions with a concentration range of 0.150 to $0.780 \mathrm{~g} / \mathrm{mL}$. Sonicated solution ( 5 to $10 \mu \mathrm{L}$ ) was deposited onto $510^{\circ} \mathrm{C}$ Curie-point pyrolysis wires. A temperature rise time of $100 \mathrm{~ms}$ and a final temperature hold of $9.9 \mathrm{~s}$ were used for all pyrolyses. The transfer line temperature was $325^{\circ} \mathrm{C}$. Experiments have been conducted to show that no secondary pyrolyses are caused by the heated transfer line. All dipeptides were purchased from Sigma Chemical Company (St. Louis, MO).

\section{Results and Discussion}

\section{Phenylalanyl-Leucine}

The pyrolysis mass spectrum of phenylalanyl-leucine (PHE-LEU), 6, shown in Figure 1, has a highest mass peak at $M^{+}-18$, which suggests a water loss. On the basis of previous literature $[3,6]$, this loss was attributed to condensation of the amino group with the hydroxy portion of the acid end to form the cyclo-dipeptide, 7 , as shown in step A of Figure 2 . The importance of the cyclo-dipeptide $(m / z 260)$ as a precursor for further fragmentation can be readily seen by comparing the full spectrum, Figure 1, with the product ion spectrum of $m / z 260$, Figure 3. Table 1 summarizes the product ions of all pertinent peaks in the PHE-LEU spectrum. Except for $m / z$ 187, 120, and 103, the full spectrum and the spectrum of product ions of $\mathrm{m} / \mathrm{z} 260$ are very similar. After dehydration, either isobutene from the leucine residue or the benzyl from the phenylalanine residue is lost. This is indicated, respectively, by the loss of $56 \mathrm{u}$ to give $m / z 204,8$, (step B, Figure 2)

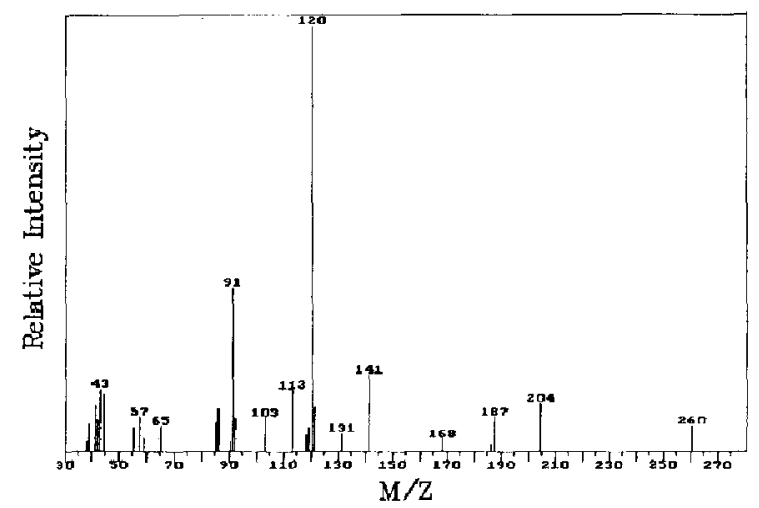

Figure 1. Pyrolysis mass spectrum of phenylalanyl-leucine. 


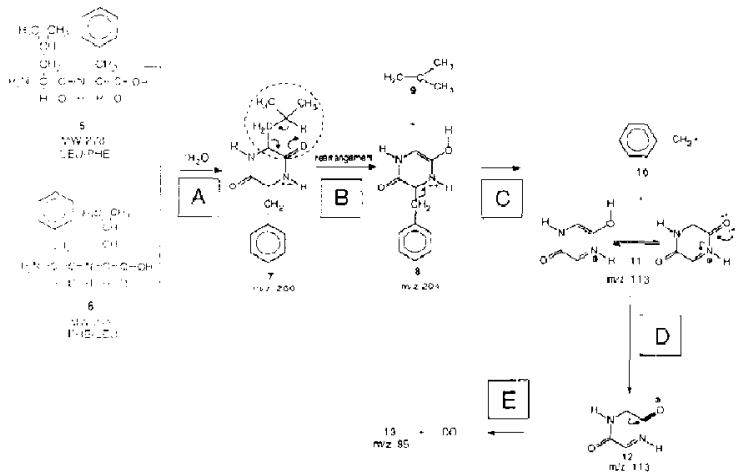

Figure 2. General fragmentation pathway 1 of phenylalanylleucine and leucyl-phenylalanine.

and by the loss of $91 \mathrm{u}$ to give $m / z 169,14$, (step $\mathrm{B}$, Figure 4). Others $[3,10]$, however, stated that the residues' side groups would remain on fragments from the collapse of the cyclo-dipeptide rather than from cleavage of the substituents before breakup of the cyclo-dipeptide.

Isobutene loss, $56 \mathrm{u}$, from the cyclo-dipeptide, $\mathrm{m} / \mathrm{z}$ 260 , is postulated to occur (step $B$ in Figure 2) by a six-membered EI-induced rearrangement. Benzyl (91 u) can then be lost from the phenylalanine residue. The product ion spectrum of $\mathrm{m} / \mathrm{z} 204$ indicates a loss of 91 u from $m / z$ 204, but no peak at $m / z$ 91. This suggests formation of a radical, but not ionic benzyl. The loss of isobutene and benzyl produces cyclic keto-enol tautomers, 11, with a characleristic peak $m / \hat{z} 113$ (step $C$ in Figure 2), which further decompose by loss of $\mathrm{CO}$ to form 13, $m / z$ 85, (steps $D$ and $E$ in Figure 2).

It is also observed that berkyl may be lost first from the cyclo-dipeptide ( $m / z 260)$ rather than isobutene. In this case, the series $m / z 169,141,113$, and 85 arises (steps $B$ and $D$ to $H$ in Figure 4). A product ion spectrum of $m / z 169,14$, shows a loss of 56 u (rearranged isobutyl) but no peak at $m / z 56$, suggesting

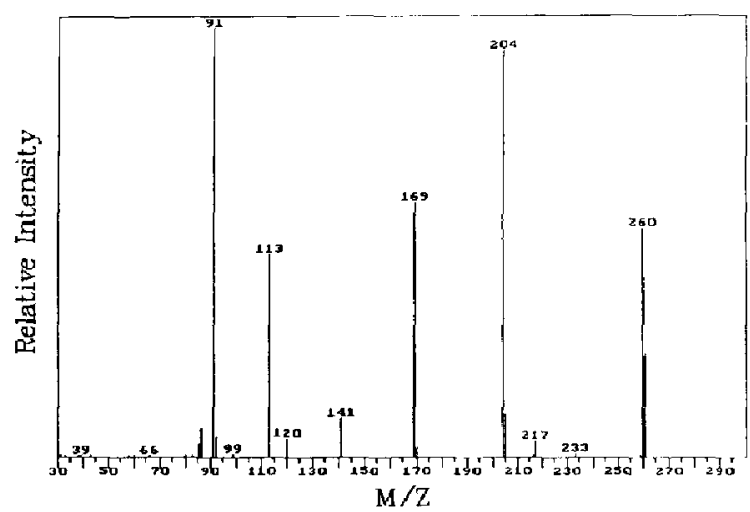

Figure 3. Pyrolysis mass spectrum of product ions of $m / 2260$ of phenylalanyl-leucine.
Table 1. Product ions of major peaks in the PHE-LEU spectrum

\begin{tabular}{cc}
$\begin{array}{c}\text { Precursor } \\
\text { ior }{ }^{a}(\mathrm{~m} / \mathrm{L})\end{array}$ & \begin{tabular}{c} 
Prouduct ions $(\mathrm{m} / \mathrm{h})$ \\
\hline 260
\end{tabular} \\
204 & $204,169,141,120,113,91,86$ \\
187 & 113,85 \\
169 & $141,113,85$ \\
141 & $113,113,85$ \\
120 & 103 \\
113 & 85 \\
103 & 77 \\
91 & 65 \\
86 & 43 \\
\hline
\end{tabular}

an ion that generates product ions is referred to as a precursor ian

retention of the charge on the ring (step $\mathrm{C}$ of Figure 4) to give $m / z$ 113. Isobutene can also be lost in several steps involving hydrogen and methyl migrations (steps $\mathrm{D}$ to $\mathrm{H}$ of Figure 4), which account for $m / z 141,15$.

The peak at $m / z 187$ (Figure 1) results from the loss of a benzyl from an unstable dipeptide molecular ion $(\mathrm{m} / \mathrm{z} 278)$. Two undefined successive losses from $\mathrm{m} / \mathrm{z}$ 187 produce $m / z 141$ and 113 . Product ion spectra of both $m / z 187$ and 141 show peaks at $m / z 113$ and 85, which clearly indicate that $m / z 187$ and 141 are precursors for the cyclic keto-enol tautomers $(\mathrm{m} / \mathrm{z} 113)$.

Cleavage along the peptide backbone also occurs; in fact, this is largely responsible (step A, Figure 5) for the base peak, $m / z .120$, in the full spectrum (Figure 1). The product ion spectrum of $m / z 120,16^{\prime}$, shows $m / z$ 103,17 , which suggests ammonia loss (step C, Figure 5 ), and $m / z 77,18$, loss of acetylene (step $D$, Figure 5). The other source of $\mathrm{m} / \mathrm{z} 120$ is the cyclo-dipeptide $(\mathrm{m} / \mathrm{z}$ 260). Breakup of the cyclo-dipeptide occurs as shown for 2 in reaction 1 where $R_{1}=$ phenyl and $R_{2}=$ isobutyl. A spectrum of precursor ions of $m / z \quad 120$

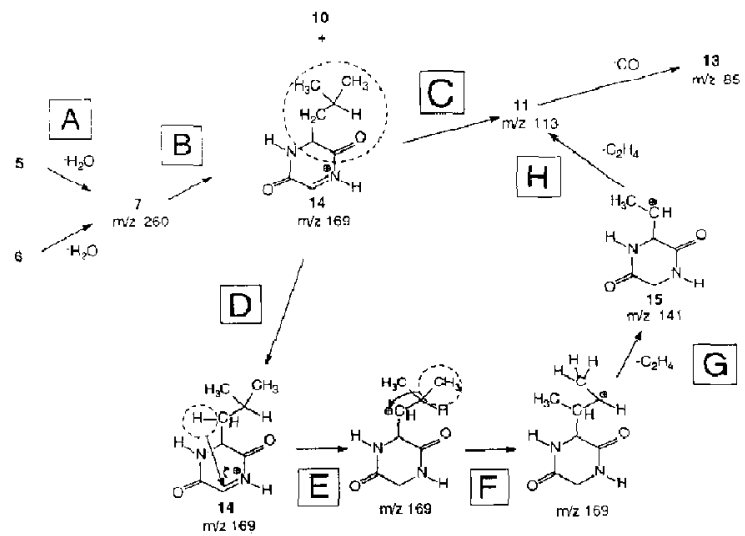

Figure 4. General fragmentation pathway 2 of phenylalanylleucine and leucyl-phenylalanine. 


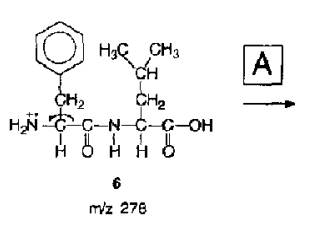

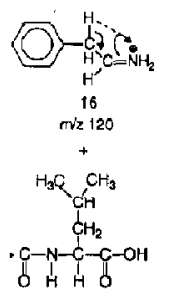

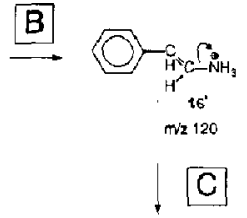

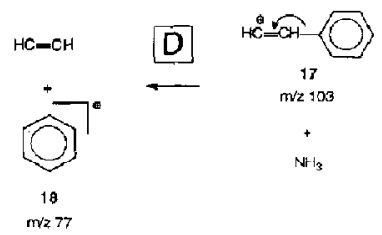

Figure 5. Fragmentation pathway 3 of phenylalanyl-leucine.

shows that the dipeptide, $6(\mathrm{~m} / \mathrm{z} 278)$ and the cyclo-dipeptide, $7,(\mathrm{~m} / \mathrm{z} 260)$ are both precursors to $\mathrm{m} / \mathrm{z} 120$.

\section{Leucyl-Phenylalanine}

A comparison of the pyrolysis mass spectrum of leucyl-phenylalanine (LEU-PHE), 5, Figure 6, with the corresponding spectrum of PHE-LEU, Figure 1, shows them to be similar with only two significant differences between the two spectra. One difference is the strong changes in the relative intensities of $m / z 86$ and 120 from PHE-LEU (Figure 1) to LEU-PHE (Figure 6). The base peak for LEU-PHE, $m / z 86$, is lower in PHE-LEU, while the base peak for PHE-LEU, $m / z 120$, is lower in LEU-PHE. The other difference between the spectra is the absence of $m / z 187$ from the LEU-PIE spectrum (Figure 6). These differences carl be attributed to the change in amino acid sequence.

In particular, the $\mathrm{N}$-terminal amino acid determines the base peak in the spectra of both PHE-LEU and LEU-PHE. It was noted in the discussion of PIE-LEU

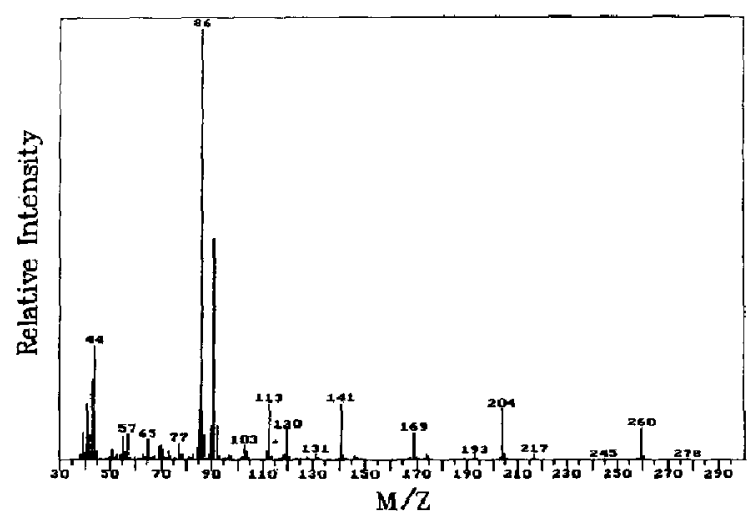

Figure 6. Pyrolysis mass spectrum of leucyl-phenylalanine.

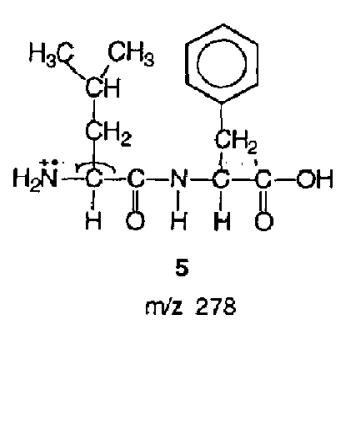<smiles>CC(C)CC=[NH2+]</smiles>

19

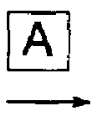

$\mathrm{m} / 286$

Figure 7. Fragmentation pathway 4 of leucyl-phenylalanine.

that cleavage of the dipeptide yields the $\mathrm{N}$-terminal product with a mass $m / z 120$ (Figure 5). The same type of cleavage in LEU-PHE (Figure 7) produces 19, $\mathrm{m} / \mathrm{z}$ 86. There are three postulated reasons why $m / z 86$ is predominantly a product ion of the dipeptide molecular ion. First, a precursor ion spectrum of $m / z 86$ shows the cyclo-dipeptide and its fragments to be an insignificant source, relative to the molecular ion. Second, in the product ion spectrum of $m / z 260$ (Figure 8), the cyclo-dipeptide, $m / z 86$, is one of the smallest peaks. It is certainly not strong enough to be the base peak of the full LEU-PHE spectrum (Figure 6). Third, Table 2 readily shows that for the product ions of all major ions from $m / z 260$ to $91, m / z 86$ appears as a product ion in only one spectrum.

Another consequence of residue sequence is the absence of $m / z 187$ from the LEU-PHE spectrum (Figure 6). Ionization and cleavage of the dipeptide are known to occur on the $\mathrm{N}$-terminal amino acid [3] (Figure 5), but the phenylalanine residue of LEU-PHE is at the C-terminal; thus, $m / z \quad 187$ would not be expected in the LEU-PHE spectrum (Figure 6).

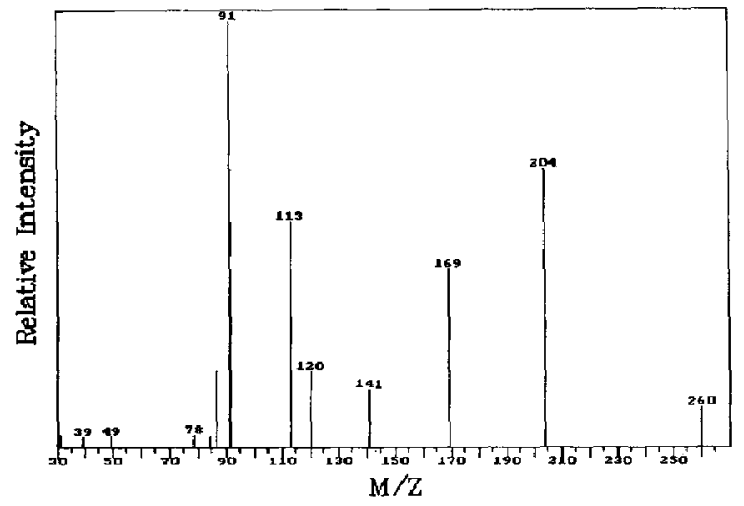

Figure 8. Pyrolysis mass spectrum of product ions of $m / 2260$ of leucyl-phenylalanine. 
Table 2. Product ions of major peaks in the LEU-PHE spectrum

\begin{tabular}{cc}
$\begin{array}{c}\text { Precursor } \\
\text { ion }(m / s)\end{array}$ & Producl ions $(\mathrm{m} / \mathrm{L})$ \\
\hline \hline 260 & $204,169,141,120,113,91,86$ \\
204 & 113,85 \\
169 & $141,113,85$ \\
141 & 113,85 \\
120 & 103 \\
113 & 85 \\
103 & 77 \\
91 & 65 \\
86 & 43 \\
\hline
\end{tabular}

In addition to cleavage of the dipeptide, dehydration and cyclization occur in LEU-PHE. The resulting cyclo-dipeptide undergoes three further fragmentation pathways that have been previously discussed for PHE-LEU. These mechanisms give rise to the same three series of peaks in the spectra of both PHE-LEU (Figure 1) and LEU-PHE (Figure 6):

$$
\begin{aligned}
& m / z 260 \rightarrow 204 \rightarrow 113 \rightarrow 85 \text { (Figure } 2) \\
& m / z 260 \rightarrow 169 \rightarrow 113 \rightarrow 85 \text { (Figure } 4) \\
& m / z 260 \rightarrow 141 \rightarrow 113 \rightarrow 85 \text { (Figure } 4)
\end{aligned}
$$

\section{Methionyl-Leucine}

Methionyl-leucine (MET-LEU), 20, (Figure 9) undergoes the same two general fragmentations, cleavage of the dipeptide and cleavage of a cyclo-dipeptide, that have been discussed for PHE-LEU and LEU-PHE. The MET-LEU pyrolysis mass spectrum (Figure 10) shows the molecular ion, $m / z 262$, and its related $\mathrm{N}$-terminal cleavage product $m / z$ 104, plus the cyclo-dipeptide ion, $m / z 244$, and its related cleavage product $m / z 113$. Cleavage of the $\mathrm{N}$-terminal methionine residue (Figure 9) generates the MET-LEU base peak, $m / z$ 104 (Figure 10 ). This is supported by the following: the presence of

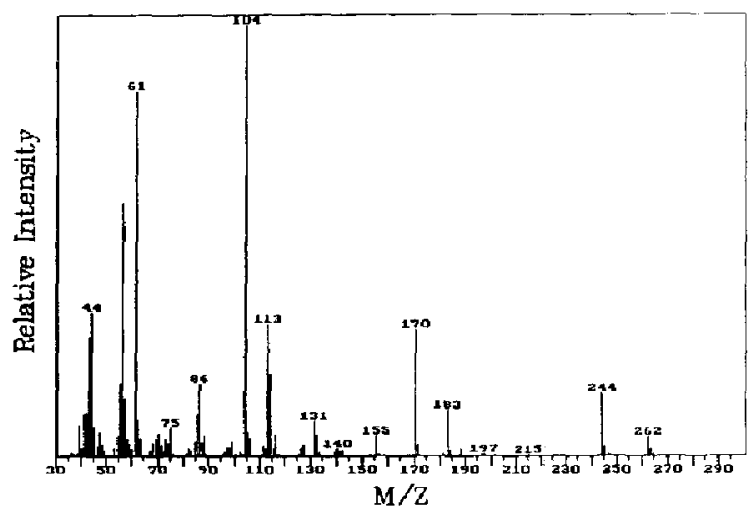

Figure 9. Pyrolysis mass spectrum of methionyl-leucine.

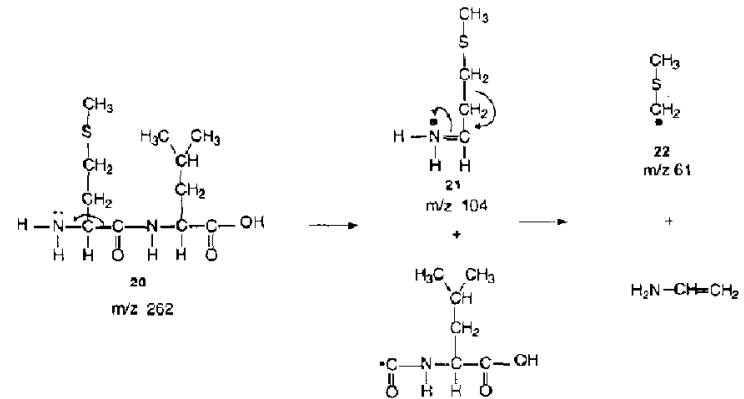

Figure 10. Fragmentation pathway 5 of methionyl-leucine.

$m / z 61,22$, which is a characteristic peak of methionine in the product ion spectrum of $m / z 104,21$; the presence of a strong $\mathrm{m} / \mathrm{z} 104$ peak in the product ion spectrum of $m / z 262,20$, the dipeptide molecular ion; and the absence of $m / 2104$ in the product ion spectrum of $\mathrm{m} / \mathrm{z}$ 244, the cyclo-dipeptide. Relevant METLEU product ions are listed in Table 3.

Cleavage of the rycln-dipeptide, 23, can occur by the initial loss of ethenyl methyl sulfide, 24, from the methionyl residue (step $A$ in Figures 11 and 12) by an EI-induced six-membered $\gamma$-hydrogen rearrangement to give $25, m / z 170$, or by radical cleavage of methyl methylene sulfide, 30 , from the methionine residue to give 31, $m / z 18.3$ (Figure 13). If ethenyl methyl sulfide, $74 \mathrm{u}$, is lost first by the six-membered $\gamma$-hydrogen rearrangement, then loss of isobutene from the leucine residue occurs next also by $y$-hydrogen rearrangement (see product ions of $m / z 170$ ) to give $27, m / z 114$ (step $\mathrm{B}$, Figure 11). This is followed by the loss of $\mathrm{CO}$ to give 28. Isobutyl may he lost instead of isobutene. This can occur by radical cleavage to give $m / z 113$ (step B, Figure 12). On the other hand, if methyl methylene sulfide is cleaved first, $61 \mathrm{u}$, from the cyclo-dipeptide (step A, Figure 13), it is immediately followed by two successive ethylene losses to produce $32, \mathrm{~m} / \mathrm{z} 155$ and 33, $m / z 127$ (steps $\mathrm{E}$ and $\mathrm{F}$ in Figure 13).

Isobutene loss can occur before the cleavage of the methionine group. In this case 34, $m / z 188$ (Figure 14) arises followed by a rearrangement loss of the me-

Table 3. Product ions of major peaks in the MET-LEU spectrum

\begin{tabular}{cc}
$\begin{array}{c}\text { Precursor } \\
\text { ion }(\mathrm{m} / \mathrm{z})\end{array}$ \\
\hline 262 & $197,183,170,155,114,113,62$ \\
244 & $155,127,86$ \\
183 & $114,113,86,85,57$ \\
170 & $116,88,86$ \\
131 & $86,85,57$ \\
114 & 85,57 \\
113 & 61,56 \\
104 &
\end{tabular}




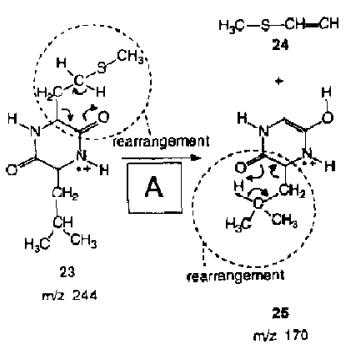

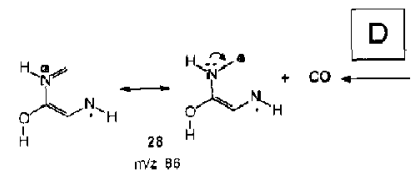

Figure 11. General fragmentation pathway 1 of methionylletcine and leucyl-methionine.

thione residue to give the keto-enol tautomers $11, m / z$ 113.

\section{Leucyl-Methionine}

The base peaks for LEU-MET and MET-LEU, as with PHE-LEU and LEU-PHE, are predominantly delermined by the $\mathrm{N}$-terminal residue. This is clearly shown by the intensities of $m / z \quad 86$ and 104 in the spectra of LEU-MET and MET-LEU shown in Figures 10 and 15. The N-terminal leucine residue of LEU-MET cleaves in a similar way as previously shown for LEU-PHE in Figure 7 .

In all other respects, the LEU-MET and MET-LEU spectra are extremely similar. Even the relative intensities of the major peaks (excluding $m / x$ 86, 104, and 131) are essentially the same. The product ions of these peaks shown in Table 4 provide evidence that they are produced by the same fragmentations of the cyclo-dipeptide as discussed for MET-LEU (see Figures 11 to 14).
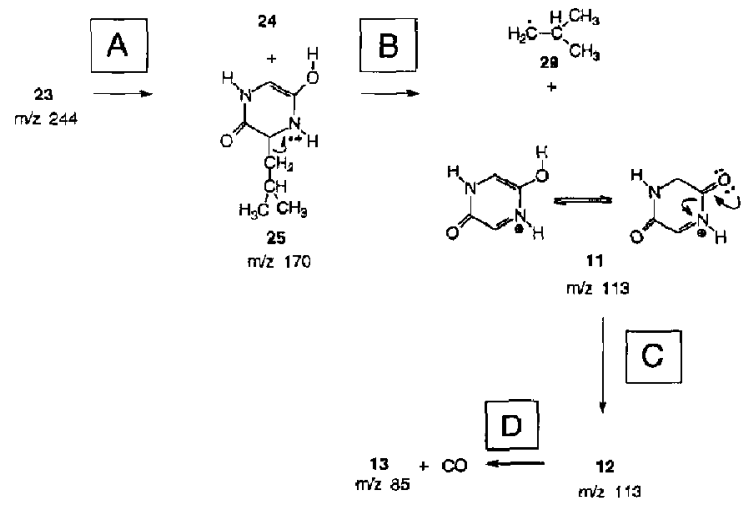

Figure 12. General fragmentation pathway 2 of methionylleucine and leucyl-methionine.

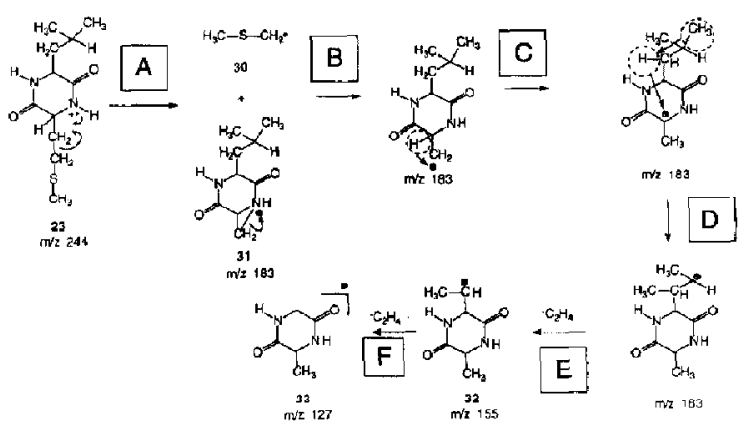

Figure 13. Fragmentation pathway 3 of methionyl-leucine and leucyl-methionine.

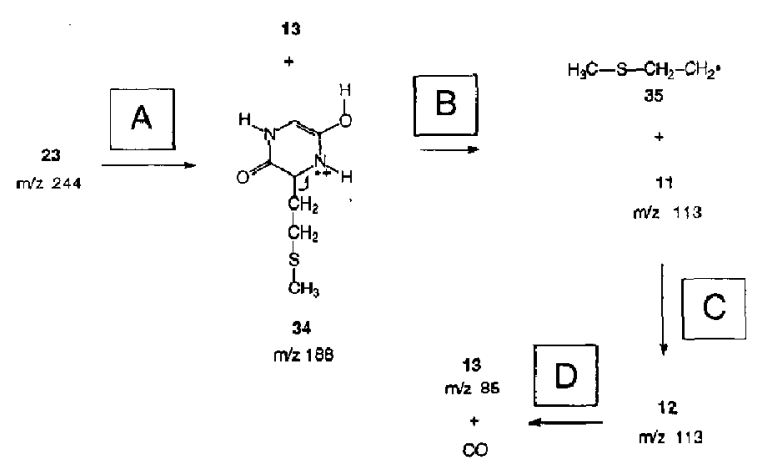

Figure 14. Fragmentation pathway 4 of methionyl-leucine and leucyl-methionine.

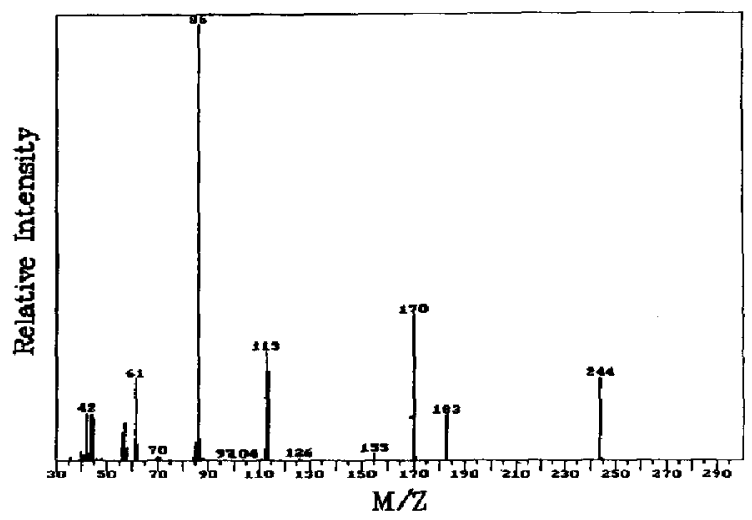

Figure 15. Pyrolysis mass spectrum of leucyl-methionine.

Table 4. Product ions of major peaks in the LEU-MET spectrum

\begin{tabular}{cc}
\hline $\begin{array}{c}\text { Precursor } \\
\text { ion }(m / z)\end{array}$ & Product ions $(m / z)$ \\
\hline 244 & $197.183 .170 .155 .114,113.62$ \\
183 & $155,127,86$ \\
170 & $114,113,85,57$ \\
114 & $86,85,57$ \\
113 & 85,57 \\
86 & 44 \\
\hline
\end{tabular}


Table 5. Major peaks in spectra of several other dipeptides containing polar and nonpolar side groups

\begin{tabular}{lcc}
\hline Dipeptides & MW $(u)$ & Major Peaks $(m / 2)$ \\
\hline Alanyl-phenylalanine & 236 & $218,127,99,91,71$ \\
Valyl-methionine & 248 & $248,230,187,169,156,141,113,85,72,61$ \\
Methionyl-valine & 248 & $248,230,187,169,156,141,113,85,72,61$ \\
Methionyl-serine & 236 & $236,218,157,144,131,114,113,85,75,61$ \\
Seryl-methionine & 236 & $218,157,144,126,114,113,85,75,61$ \\
Methionyl-glycine & 206 & $206,188,131,114,104,85,76,61$ \\
\hline
\end{tabular}

\section{Other Vipeptides}

Several other dipeptides fragment by the same pathways previously discussed for PIIL-LEU, LEU-PIIL, MET-LEU, and LEU-MET. This is summarized in Table 5, which lists the major peaks above $\mathrm{m} / \mathrm{z} 60$ that appeared for the corresponding dipeptide in a scan range $m / z 35$ to 350 . The dipeptides containing methionine all have three sets of peaks in common: peaks general to their cyclo-dipeptides $\left(\mathrm{M}^{+}-18, \mathrm{~m} / \mathrm{z} 114\right.$ or 113 , and $m / z$ 85), peaks from rearrangement loss or cleavage of specific amino acid residues (for example, $m / z 61$ from methionine), and peaks from N-terminal cleavage. It is interesting to note that product ion spectra (not shown) for alanyl-phenylalanine reveal that the series $m / z \quad 218,127$, and 99 (Table 5) corresponds to the cyclo-dipeptide peaks $\mathrm{M}_{.}^{+}-18,113$, and 85 for the methionine dipeptides. This suggests that the methyl group from the alanine residue is retained on the cyclo-dipeptide, but the benzyl group from the phenylalanine residue is lost. Tandem mass spectra of alanyl-alanine and alanyl-glycine were also cxamined. They were found to support the mechanisms determined by Junk and Svec [4].

\section{Conclusions}

The data from the Py-MS of 12 dipeptides clearly established two levels of general electron ionization and thermal fragmentations for dipeptides. On one level, either direct dehydration of the dipeptide to form cyclo-dipeptide or cleavage of the dipeptide ocrurs. Although these cleavages have been reported by others, it is important to note that the present study shows that their formation occurs simultaneously and rapidly under the conditions of our experiments. This contrasts with Svec and Junk [3] who found primarily cyclo-dipeptide formation and cleavage for the first 45 min of their experiment, and then dipeptide cleavage for the remaining several hours.

The other level of fragmentation involves reactions of the side group in one of three ways: simple cleavage of a side group to form a stable product (for example, benzyl from a phenylalanine residue); cleavage of a side group with migration or rearrangement, especially by a six-membered rearrangement if there is a $\gamma$-hydrogen relative to a carbonyl group (for example, isobutene from a leucine residue); or no cleavage of a side group if it is aliphatic with three or fewer carbons (for example, methyl in an alanine residue). Previously $[3,10]$ the loss of the side chain had been postulated to occur atter the breakup of the cyclo-dipeptide, as in reaction 1 . The cleavage of the side chain before fragmentation of the cyclo-dipeptide was not observed in the earlier studies [3] because the dipeptides studied contained no side chain (GLY-GLY) or an aliphatic one with less than three carbons (ALA-ALA).

A problem associated with Py-MS results is the inability to differentiate between the ions due to pyrolysis products and those from electron ionization fragmentations. With the exception of the dehydration product, the tandem mass spectrometry results have shown that the ions in this study are a result of electron ionization. Resolution of the genesis of the dipeptide dehydration product will have to be determined by using the same experimental approach with a soft ionization technique.

\section{Acknowledgments}

The authors thank Dr. E. W. Sarver for helpful discussions and Teledyne CME for financial support.

\section{References}

1. Merritt, C. Jr.; Robertson, D. H. J. Gas Chromatogr. 1967, 5, 96-98.

2. Smith, G. G.; Reddy, G. S.; Boon, J. J. J. Chem. Soc. Perkin Trans. II 1988, 203-211.

3. Svec, H. J.; Junk, G. A. J. Am. Chem. Soc. 1964, 86, 2278-2282

4. Junk, G. A.; Svec, H. J. Anal. Biochem. 1963, 6, 199-202.

5. Biemalu, K. Mass Spectromelry; Organi- Chemical Applicalions; McGraw-Hill: New York, 1962; pp 283-296.

6. Budzikiewicz, H.; Djerassi, C.; Williams, D. H. Structure Elucidation of Natural Products by Mass Spectrometry. Vol. II; Holden-Day: San Francisco, 1964; pp 183-202.

7. Advanced Methods in Protein Sequence Deterntination; Needleman, S. B., Ed.; Springer-Verlag: Berlin, 1977; p 130.

8. Gross, D.; Grodsky, G. J. Am. Chem. Soc. 1955, 77, 1678-1680.

9. Hurd, C. D. The Pyrolysis of Carbon Compounds; The Chemical Catalog Co.: New York, 1929; pp 462-465.

10. Ratcliff, M. A. Jr.; Medley, E. E: Simmonds, P. G. J. Org. Chem. 1974, 39, 1481-1490.

11. Lubman, D. M.; Li Liang Appl. Spec. 1989, 43, 543-548.

12. New Techniques in Amino Acti, Peptide, Protein Analysis; Niederwieser, A., Ed.; Ann Arbor Science: Ann Arbor, MI, 1971; pp 179-180.

13. Deluca, S.; Sarver, E. W; Harrington, P. de B.; Voorhees, K. J. Anal. Chem. 1990, 62, 1465. 